\title{
Association between pelvic exenteration and hyperthermic intraperitoneal chemotherapy for rectal cancer
}

\author{
Nicolae Bacalbasa ${ }^{1,2}$, Irina Balescu ${ }^{3}$, Adnan Al Aloul ${ }^{4,5}$ \\ ${ }^{1}$ Department of Visceral Surgery, Center of Excellence in Translational Medicine, \\ Fundeni Clinical Institute, Bucharest, Romania \\ ${ }^{2}$ Department of Obstetrics and Gynecology, \\ "Carol Davila" University of Medicine and Pharmacy, Bucharest, Romania \\ ${ }^{3}$ Department of Surgery, Ponderas Academic Hospital, Bucharest, Romania \\ ${ }^{4}$ Department of Surgery, Ramnicu Sarat County Hospital, Buzau, Romania \\ ${ }^{5}$ Doctoral School, "Titu Maiorescu“ University, Bucharest, Romania
}

\begin{abstract}
Colorectal cancer spreads via multiple pathways, the most commonly encountered ways being represented by the peritoneal and hematogenous ones as well as by direct contiguity. At this time significant benefits in terms of survival have been reported in cases presenting locally advanced lesions and for those presenting extended peritoneal lesions of peritoneal carcinomatosis especially if radical resection is achievable. In this respect, certain authors went further and investigated whether the association of the two surgical strategies extended pelvic resections and cytoreductive surgery of the peritoneal metastases could improve survival in cases in which both patterns of spread are present. Meanwhile, the possibility of association of hyperthermic intraperitoneal chemotherapy (HIPEC) was also investigated. The aim of the current study is to review and analyze the most relevant studies conducted on this issue.
\end{abstract}

Keywords: rectal cancer, pelvic exenteration, hyperthermic intraperitoneal chemotherapy, morbidity, survival

\section{INTRODUCTION}

Colorectal cancer represents one of the most commonly encountered digestive malignancies which is usually diagnosed in early stages of the disease due to the fact that is frequently associated with severe clinical signs such as constipation or association between constipation and diarrhea, pelvic pain, rectal bleeding and weight loss. However, in certain cases these symptoms are frequently neglected by the patient and the diagnostic is finally established when local dissemination or invasion of the surrounding organs already occurs (1).

\section{PATTERNSOFSPREADINRECTALCANCER}

When it comes to the possibility of local invasion, whenever malignant cells destroy the natural barriers at this level represented by the serosal surface, invasion of the surrounding organs occur leading to the apparition of locally advanced pelvic tumors; in such cases extended multivisceral resections are needed in order to control the disease. In the meantime whenever malignant cells destroy the serosal seeding can occur at the level of the peritoneum and therefore disseminated lesions of peritoneal carcinomatosis develop (2-4). Initially these cases have been considered as having distant metastases and therefore were submitted to palliative oncological therapies (5). However, it should not be omitted the fact that in such cases lesions occur via loco-regional spread and not through a systemic route; in this respect certain authors consider that cases presenting locally invasive rectal cancer in association with peritoneal nodules have an abdominal confined disease and 
therefore consider that they can be submitted to radical surgery with curative intent $(6,7)$. The aim of the current paper is to review the most relevant studies conducted on the issue of the possibility of association between cytoreductive surgery, HIPEC and pelvic exenteration.

\section{RATIONALE FOR ASSOCIATION BETWEEN CYTOREDUCTIVE SURGERY AND PELVIC EXENTERATION}

In order to maximize the effects of debulking surgery in cases presenting both peritoneal metastases and local invasion, association between extended pelvic resections (such as total or posterior pelvic exenteration) and cytoreductive surgery including maximal resection of the peritoneal nodules of carcinomatosis has been recently investigated $(6,7)$. However, it should not be omitted the fact that each procedure in part is associated with significant risks of postoperative complications $(8,9)$. Therefore, it is estimated that the postoperative morbidity rates after cytoreductive surgery in the setting of colorectal cancer range between 10\% and $65 \%$, while the risks of postoperative complications after pelvic exenteration can also reach $50 \%(8-12)$. Due to this reason, the initial trend was to exclude from surgery cases presenting the association of these two entities and to rather consider that these patients should be referred for palliative systemic treatment.

Meanwhile, it has been observed that cases with a favorable postoperative evolution might report a significant benefit in terms of survival which might surpass the value of $30 \%$ at a 5 year follow up $(8,9,12)$. In this respect attention was focused on the possibility of association of the two methods, on identifying the most significant prognostic factors and on creating of a valid protocol which should be able to identify the cases which might benefit most from this aggressive therapeutic strategy. As expected, initially this subject was reported as isolated situations and presented as case reports or case series $(6,13,14)$.

The first study which aimed to answer to the question whether such an aggressive surgical approach is justified comes from the Indian study group conducted by Shinde et al; in the paper which was published in 2019 the authors included three cases diagnosed with locally advanced rectal cancer and peritoneal metastases which were successfully submitted to this combined approach (6).

One of the most relevant studies which were conducted on this issue has been recently pub- lished by Tuech et al. and was published in Cancers in 2020. The study included 319 patients submitted to cytoreductive surgery and HIPEC from 2006 to 2020 , among them 16 cases being submitted to a synchronous pelvic exenteration; the main indications were represented by locally advanced rectal cancer in six cases, recurrent rectal cancer in nine cases and appendiceal cancer with carcinomatosis in one case. The median value of the peritoneal carcinomatosis index was 8 while the median number of region affected of peritoneal carcinomatosis was 3 , both values being obtained after excluding the pelvic region. In all cases complete cytoreduction was reported; when it comes to the completeness of resection at the level of the pelvic area, six out of the 16 patients were submitted to a laterally extended pelvic exenteration in order to achieve negative resection margins; even though positive resection margins were encountered in three cases submitted to extended pelvic resections, the level of positive resection margins being represented by the pre-sacral fascia. When it comes to the postoperative follow up, severe complications (defined as Dindo Clavien grade III-V complications) were reported in $56.2 \%$ of cases while two cases died during the first month postoperatively, the most severe surgical complications being represented by pelvic abscesses and urosepsis. As for the long term outcomes seven patients died during the follow up period, in six cases the death being correlated with the progression of the neoplastic disease. Therefore, the authors concluded that, in selected cases the association of these two surgical procedures might improve the long term outcomes and underlined the fact that this association should not be routinely avoided especially if resection with negative margins and complete cytoreduction are achievable (7).

Another similar study was conducted by Brown et al. and published in 2020; the study included six cases submitted to cytoreductive surgery in association with HIPEC and pelvic exenteration for primary rectal cancer (in three cases), recurrent sigmoidian cancer (in two cases) and recurrent rectal cancer (in one case). In these cases the median peritoneal carcinomatosis index was six, in all patients complete cytoreduction being achieved. Similarly to Tuech et al. study, the rate of severe postoperative complications (defined as Dindo-Cavien grade III-IV complications) was 50\%; however no postoperative death was reported during the first postoperative month. As for the long term outcomes, at a median follow up of 11,5 months three patients were alive with no recurrent 
disease, one patient developed an isolated pelvic recurrence, one patient developed pelvic and peritoneal recurrences while the last case developed bone metastases. Among the cases which developed recurrent disease, at the end of the follow up period two patients died of disease. Therefore the authors underlined the fact that in selected cases significant benefits in terms of survival are achievable; however they underlined once again the necessity of an adequate selection of the cases, this aspect being demonstrated through the fact that during the study period in the same institution 235 exenterative procedures and 190 cytoreductive procedures were performed, only six of these cases being considered as suitable for a combined approach (15).

Conflict of interest: none declared

Financial support: none declared

\section{REFERENCES}

1. Siegel RL, Miller KD, Jemal A. Cancer statistics, 2019, Ca-Cancer. J Clin. 2019;69:7-34.

2. Strelez C, Chilakala S, Ghaffarian K, Lau R, Spiller E, Ung N, Hixon $D$, et al. Human colorectal cancer-on-chip model to study the microenvironmental influence on early metastatic spread. iScience. 2021;24(5):1-24.

3. Hachey SJ, Movsesyan S, Nguyen QH, Burton-Sojo G, Tankazyan A, et al. An in vitro vascularized micro-tumor model of human colorectal cancer recapitulates in vivo responses to standard-of-care therapy. Lab Chip. 2021;21:1333.

4. Woodford-Richens KL, Rowan AJ, Gorman P, Halford S, Bicknell DC, Wasan HS, Roylance RR, Bodmer WF, Tomlinson IP. SMAD4 mutations in colorectal cancer probably occur before chromosomal instability, but after divergence of the microsatellite instability pathway, Proc Natl Acad Sci U S A. 2001;98:9719-9723.

5. Cassidy J, Clarke S, Diaz-Rubio E, Scheithauer W, Figer A, Wong R, et al. Randomized Phase III Study of Capecitabine Plus Oxaliplatin Compared With Fluorouracil/Folinic Acid Plus Oxaliplatin As First-Line Therapy for Metastatic Colorectal Cancer. J Clin Oncol. 2008;26:2006-2012.

6. Shinde, RS, Acharya R, Kumar NA Solanki S, Desouza A, Saklani A. Pelvic Exenteration with Cytoreductive Surgery and Hyperthermic Intraperitoneal Chemotherapy (CRS + HIPEC) for Rectal Cancer Case Series with Review of Literature. Indian J Surg Oncol. 2019;10:80-83

7. Tuech JJ, Pinson J, Nouhaud FX, Wood G, Clavier T, Sabourin JC, Di Fiore F, Monge M, Papet E, Coget J. Total Pelvic Exenteration, Cytoreductive Surgery, and Hyperthermic Intraperitoneal Chemotherapy for Rectal Cancer with Associate Peritoneal Metastases: Surgical Strategies to Optimize Safety. Cancers. 2020;12:3478.

\section{CONCLUSIONS}

Although initially considered as candidates for palliative chemotherapy solely, patients with peritoneal carcinomatosis and locally invasive rectal cancer seem to benefit from a combined approach consisting of cytoreductive surgery, HIPEC and pelvic exenteration especially if complete cytoreduction and negative resection margins are achievable. However, it should not be omitted the fact that until now only scarce number of cases or case series have been reported. Therefore, the method is not yet well standardized, larger, prospective studies being needed in order to establish which cases could benefit most from this combined and aggressive surgical approach.

8. Chua TC, Saxena A, Schellekens JF, Liauw W, Yan TD, Fransi S, Zhao J, Morris DL. Morbidity and mortality outcomes of cytoreductive surgery and perioperative intraperitoneal chemotherapy at a single tertiary institution: Towards a new perspective of this treatment. Ann Surg. 2010;251:101-106.

9. Verwaal VJ, van Tinteren H, Ruth SV, Zoetmulder FA. Toxicity of cytoreductive surgery and hyperthermic intra-peritoneal chemotherapy. J Surg Oncol. 2004;85:61-67.

10. Tuech JJ, Lefebure B, Michot F, Teniere P. Pelvic exenteration for cancer. J Chir. 2005;142:367-374.

11. Tuech JJ, Schwarz L, Coget J, Bridoux V. Difficult hemostasis during radical pelvic surgery. J Visc Surg. 2015;152:119-124.

12. Heriot AG, Byrne CM, Lee P, Dobbs B, Tilney H, Solomon MJ, Mackay J, Frizelle F. Extended radical resection: The choice for locally recurrent rectal cancer. Dis Colon Rectum. 2008;51:284-291.

13. Sugarbaker PH. Total pelvic exenterative surgery in patients with peritoneal metastases from appendiceal neoplasms. A case series of 2 patients. Int J Surg Case Rep. 2019;65:279-283.

14. Müller APB, Soto Ruiz JM, Leon P, Jimenez LC, Carpio Moreno CK, de Sa Ribeiro FA. Rectal tumor with perineal invasion and peritoneal carcinomatosis: pelvic exenteration followed by cytoreductive surgery and hyperthermic intraperitoneal chemotherapy - a case report. EJSO. 2021;47(2):61-62.

15. Brown KGM, Ansari N, Solomon MJ, Austin KKS, Hamilton AER, Young CJ. Pelvic exenteration combined with cytoreductive surgery and hyperthermic intraperitoneal chemotherapy for advanced primary or recurrent colorectal cancer with peritoneal metastases. Colorectal Disease. 2021;23:186-191. 